\title{
Slope Efficiency and Dynamic Range of Traveling-Wave Multiple-Quantum-Well Electroabsorption Modulators
}

\author{
Bin Liu, Jongin Shim, Yi-jen Chiu, Hsu Feng Chou, Joachim Piprek, Senior Member, IEEE, and John E. Bowers
}

\begin{abstract}
High-performance InGaAsP-InP multiple-quantumwell traveling-wave electroabsorption modulators (EAMs) have been developed for analog optical links. A high slope efficiency of $>4 / \mathrm{V}$ at $1.55 \mu \mathrm{m}$ for a 300- $\mu \mathrm{m}$-long EAM is measured. A detailed study of the nonlinearity and the spurious-free dynamic range (SFDR) is presented. By optimizing the bias voltage and the input optical power, the SFDR can be improved by 10-30 dB.
\end{abstract}

Index Terms-Analog systems, electroabsorption, intermodulation distortion, modulation transfer functions, modulators, optical links.

\section{INTRODUCTION}

$\mathbf{E}$ XTERNAL modulators have attracted increasing attention for both digital and analog fiber-optic links, since they can provide high speed, high efficiency, and other features desirable for these applications. Two types of external modulators have been extensively studied: Mach-Zehnder modulators (MZMs) [1] and electroabsorption modulators (EAMs) [2]. The EAM is a reverse biased p-i-n diode with bulk active region [2] or multiple quantum-wells (MQWs) [3] as the absorption layer. Compared to bulk absorption layers, the absorption edge in MQWs is much sharper and moves faster with reverse biased electric field. An MQW EAM has the advantages of low driving voltage, broad bandwidth, compact size, and monolithic integration capability with other devices. It has great potential as a low power consumption and low-cost external modulator for both digital and analog communications. The slope efficiency and linearity of EAMs are additional two critical parameters for analog applications. A high slope efficiency is desirable to achieve a high link gain. Nonlinearity of EAMs causes distortion and reduces the dynamic range of an radio-frequency (RF) link. In an electrooptic MZM, the transfer curve is well known as a sinusoidal function and is independent of the input optical and RF signal. Therefore, the nonlinearity can be characterized accurately, the dynamic range can be improved by linearization techniques, resulting in spurious-free dynamic range (SFDR) as high as $133 \mathrm{~dB} \cdot \mathrm{Hz}^{4 / 5}$ [4]. On the other hand, an EAM has a complicated nonlinear transfer function and a strong dependence on

Manuscript received March 13, 2003; revised July 28, 2003.

B. Liu, Y.-J. Chiu, H. F. Chou, J. Piprek, and J. E. Bowers are with the Department of Electrical and Computer Engineering, University of California, Santa Barbara, CA 93106 USA (e-mail: liu@ece.ucsb.edu).

J. Shim is with the Department of Electrical and Computer Engineering, University of California, Santa Barbara, CA 93106 USA, on leave from the Department of Electrical and Computer Engineering, Hanyang University, Kyungki-do 425-791, Korea.

Digital Object Identifier 10.1109/LPT.2003.818918

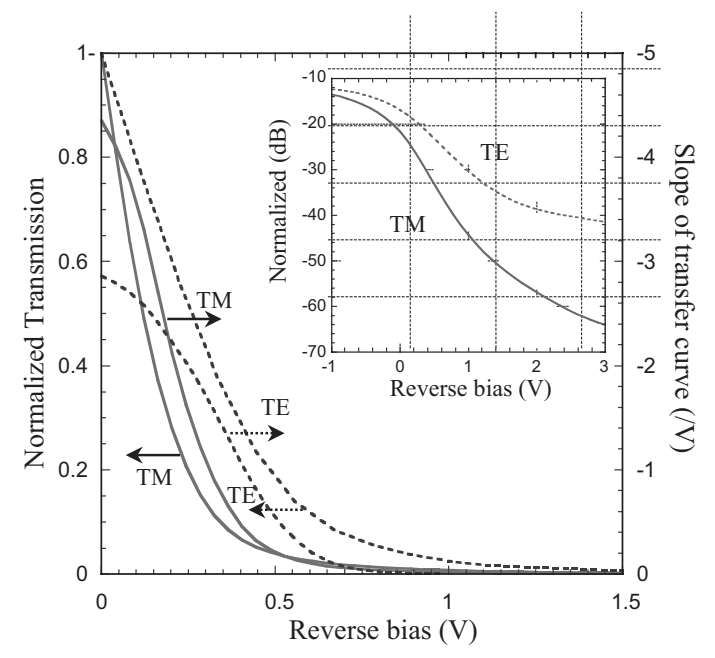

Fig. 1. Normalized transfer curve and the slope for a TW-EAM. The insert is the measured transfer curve in log scale.

input optical wavelength and power. Thus, the analysis of the nonlinearity of EAMs is essential in order to enhance the dynamic range and to achieve high-quality RF links [5].

In this letter, the analog performance of InP-based traveling-wave MQW EAMs (TW-EAM) with very low drive voltage $(<1 \mathrm{~V})$ and high slope efficiency $(>4 / \mathrm{V})$ is characterized. A detailed study of slope efficiency, nonlinearity, and SFDR of a TW-EAM is conducted.

\section{DEVICE FABRICATION AND CHARACTERISTICS}

The detailed structure and fabrication of the TW-EAM can be found in [6] and [7]. The epi-structure is an InGaAsP-based material grown by metal-organic chemical vapor deposition on a semiinsulating InP substrate. The photoluminescence peak wavelength of strain-compensation MQW active region is $1510 \mathrm{~nm}$. This is the main difference from other EAMs [6], [8], [9]. The photoluminescence peak shift will improve the slope efficiency of the EAMs at low bias.

Fig. 1 shows the typical normalized fiber-to-fiber dc transmission curve of our MQW TW-EAMs with a length of $300 \mu \mathrm{m}$ operated at a wavelength of $1555 \mathrm{~nm}$. The input optical power is $2 \mathrm{dBm}$. The dc insertion loss is about -22 and $-18 \mathrm{~dB}$, respectively, for transverse magnetic (TM) and transverse electric (TE) polarizations at $0 \mathrm{~V}$. The measured 3 -dB electrical bandwidth is larger than $25 \mathrm{GHz}$ [7]. For analog links, the slope efficiency is critical for RF gain. Fig. 1 also plots the dc slope efficiency of the normalized transfer curve. The EAM has a very 


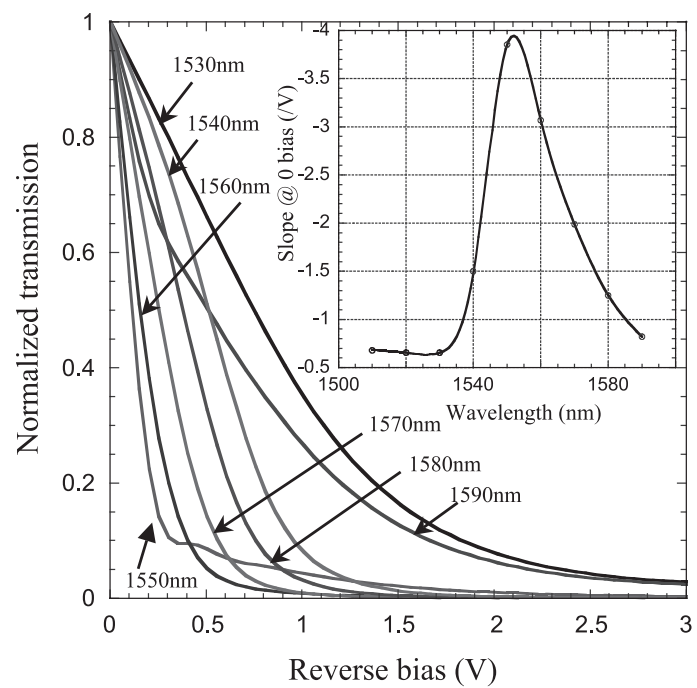

Fig. 2. Normalized transfer curve for the TM mode at different optical wavelength. The insert is the slope efficiency at zero bias.

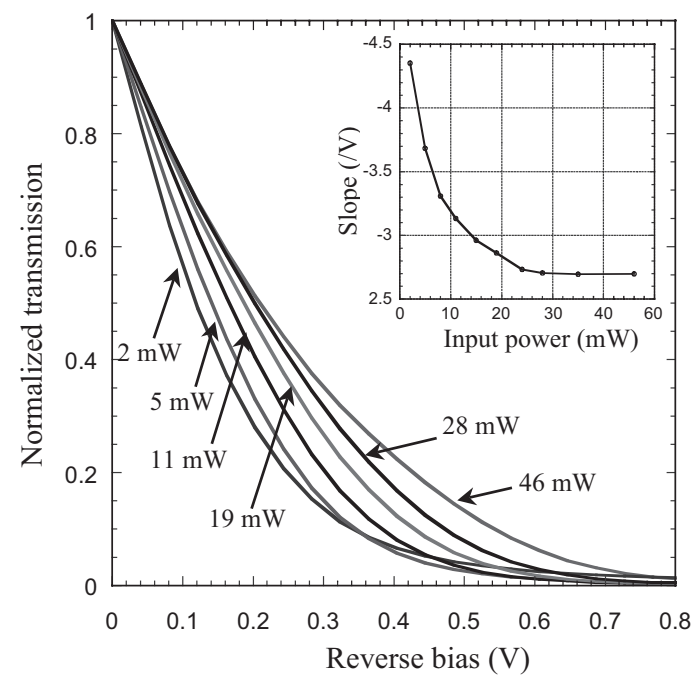

Fig. 3. Normalized transfer curve for the TM mode at different input optical power. The insert is the slope efficiency at zero bias.

high slope of $>4 / \mathrm{V}$ around 0 bias. To compare with MZMs, an equivalent voltage $V_{\pi, \text { eq }}$ can be used to express the slope efficiency of EAMs, $V_{\pi, \mathrm{eq}}=(\pi / 2)\left|\partial T / \partial V_{\mathrm{RF}}\right|^{-1}$ [5], where $T$ is the normalized transmission and $V_{\mathrm{RF}}$ is the RF voltage. Therefore, this EAM has a $V_{\pi}$ as low as $0.37 \mathrm{~V}$ with a bandwidth larger than $25 \mathrm{GHz}$.

Fig. 2 shows the normalized transfer curves at different wavelengths with the input power of $2 \mathrm{~mW}$. As we expected, MQW EAMs have different slope efficiencies and extinction ratios at different wavelengths. The dependence of the slope efficiency on wavelength for TM mode is plotted in the insert of Fig. 2. Because of this strong wavelength dependence, the linearity of EAMs can be improved by tuning the input light wavelength, and the dynamic range can be enhanced [8].

Due to carrier pile-up and band-filling effects, the input optical power also affects the performances of EAMs. Fig. 3 plots the transfer curves with different optical input power at $1553 \mathrm{~nm}$. With the increase of the input power, the slope decreases. This is a substantial difference to MZMs, which are

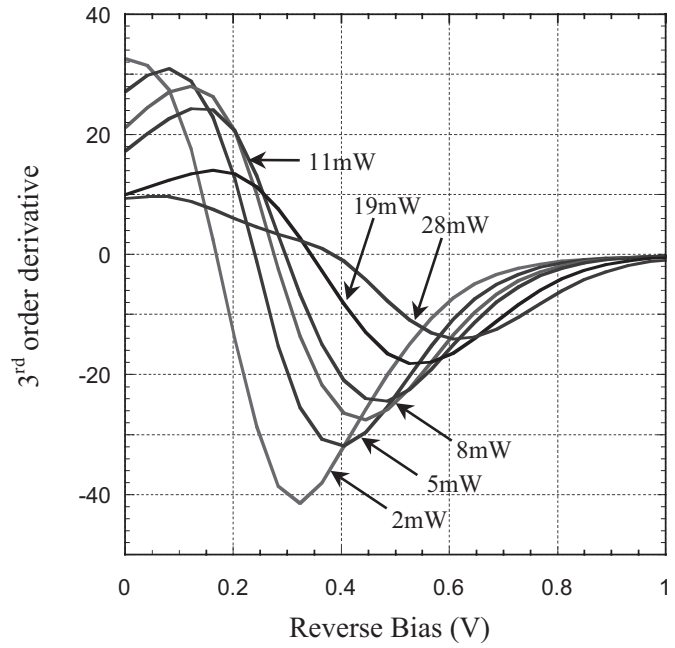

Fig. 4. Third-order derivatives of the transfer function of an EAM with different input optical power.

almost independent of optical power. Thus, the RF gain in an EAM modulated link will not be perfectly proportional to the input optical power with a slope of two.

\section{SFDR}

One of the important performance parameters for high-speed analog communication is the SFDR [4]. It is well known that the third-order intermodulation product can be minimized and a high SFDR can be achieved if a modulator is biased at the null point of the third derivative of the transfer curve [9]. Fig. 4 plots the third derivatives of the measured dc transfer functions of an MQW TW-EAM for the TM mode at different input optical powers. It is clear that there is a null point in each transfer curve and the corresponding bias depends on the input optical power. Furthermore, the third-order derivative decreases and the linearity improves with the increase of the input optical power. Thus, we expect that the third-order distortion can be minimized and a high dynamic range can be achieved by adjusting both bias voltage and the input optical power. To further improve the SFDR, the fifth-order distortion also needs to be reduced. If we check the fifth derivatives of the transfer curves, we do find that both third- and fifth-order derivatives can be simultaneously zero by carefully adjusting the input optical power and the polarization.

The two-tone method was used to investigate the suboctave SFDR of our TW-EAMs at $10 \mathrm{GHz}$. Two RF signals with frequencies 10 and $9.9 \mathrm{GHz}$ from synthesizers are combined and fed into a modulator. A high-power distributed feedback (DFB) laser with wavelength at $1553 \mathrm{~nm}$ is used as the light source.

The dynamic range is defined as the difference between the fundamental and noise output power at the point where the intermodulation power is equal to the noise floor. In this work, we calculated the noise based on the RIN of the laser source, the shot noise, and the thermal noise. The actual measurement system noise floor was limited to $-163 \mathrm{dBm} / \mathrm{Hz}$ by an electrical amplifier (7.5-dB gain at $10 \mathrm{GHz}$ ) following the photodetector. The measured suboctave SFDR results at different biases and 


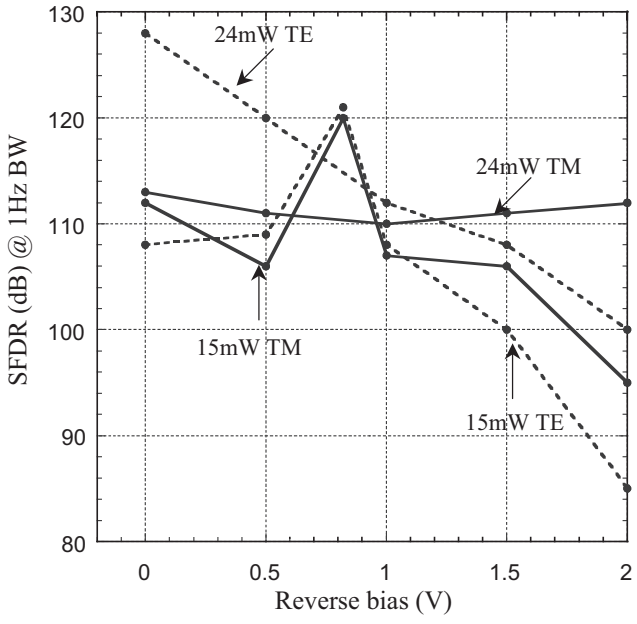

Fig. 5. Estimated SFDR based on the calculated noise floor for $1-\mathrm{Hz}$ bandwidth versus bias with different input optical powers.

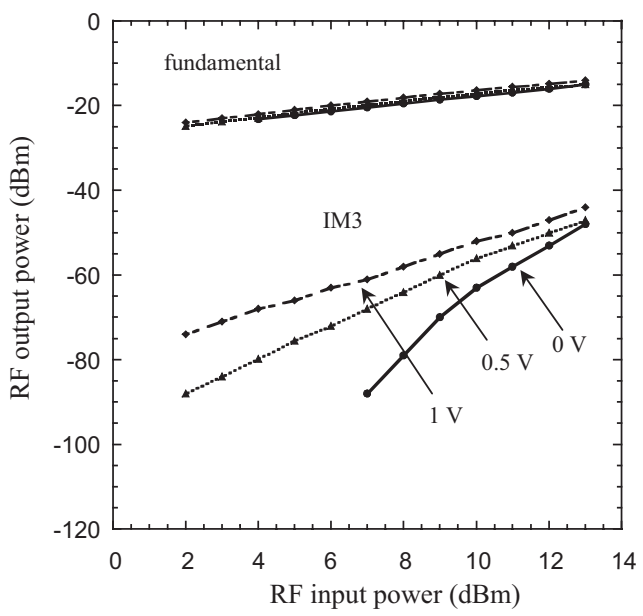

Fig. 6. Measured fundamental and third-order intermodulation signals.

different input optical powers are summarized in Fig. 5. By adjusting the bias voltage, $\sim 15-\mathrm{dB}$ dynamic range improvement can be achieved. By checking the intermodulation signals, the slope of the intermodulation product versus the input RF signal is five at the peak SFDR point, where the third-order derivative is zero. Fig. 6 shows the measured fundamental and the intermodulation RF signals at three different bias points with 24-mW TE optical input power. At 0-V bias with an average photocurrent of $0.21 \mathrm{~mA}$, the slope of the intermodulation signal is $>5$ and the highest SFDR of $128 \mathrm{~dB} \cdot \mathrm{Hz}^{4 / 5}$ is achieved when we fit the linear curve with a slope of five. (The best linear fit of the intermodulation signal has a slope of 6.5, and this increases the dynamic range by $5 \mathrm{~dB}$.) Interestingly, the intermodulation signal at this optimized point does not have a uniform slope. The slope changes from nine to five with increasing input RF power. This phenomenon has been theoretically predicted and experimentally observed in linearized electrooptic modulators [4], [10]. When the bias is increased to $1 \mathrm{~V}$, the slope of the intermodulation product is three and the corresponding SFDR is reduced to $110 \mathrm{~dB} \cdot \mathrm{Hz}^{2 / 3}$. The most interesting observation here is that the dynamic range and the third-order derivative null point strongly depend on the input optical power level, as we expected from the measured dc transfer curves. When the optical power increases from 6 to $24 \mathrm{~mW}$, the SFDR increases by $\sim 20 \mathrm{~dB}$. We also noticed that the fundamental RF power at high optical input power did not show severe reduction with increasing bias and that the optimized SFDR bias points did not match the predicted third-order zeros exactly. At this moment, we have not fully understood the physics behind this. Further theoretical and experimental investigations are needed to explain this observation.

\section{CONCLUSION}

Slope efficiency, linearity, and SFDR of MQW TW-EAMs have been investigated in detail. A high slope efficiency of $>4 / \mathrm{V}$ is measured at $1.55 \mu \mathrm{m}$ with a low input optical power for a 300- $\mu$ m-long EAM, which is equivalent to an MZM with a $V_{\pi}$ of $0.37 \mathrm{~V}$. By adjusting the bias voltage as well as the input optical power, the increase of the SFDR can be as much as $30 \mathrm{~dB}$. An SFDR as high as $128 \mathrm{~dB} \cdot \mathrm{Hz}^{4 / 5}$ at $10 \mathrm{GHz}$ has been achieved experimentally.

\section{REFERENCES}

[1] R. C. Alferness, "Waveguide electrooptic modulators," IEEE Trans. Microwave Theory Tech., vol. MIT-30, pp. 1121-1137, Aug. 1982.

[2] C. Rolland, G. Mak, K. L. Prosyk, C. M. Maritan, and N. Puertz, "High speed and low loss, bulk electroabsorption waveguide modulators at 1.3

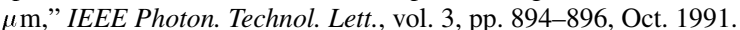

[3] D. A. B. Miller, D. S. Chemla, T. C. Damen, A. C. Gossard, W. Wiegmann, T. H. Wood, and C. A. Burus, "Band-edge electroabsorption in quantum well structures: the quantum-confined Stark effect," Phys. Rev. Lett., vol. 53, pp. 2173-2175, 1984.

[4] G. E. Betts and F. J. O'Donnell, "Microwave analog optical links using suboctave linearized modulators," IEEE Photon. Technol. Lett., vol. 8, pp. 1273-1275, Sept. 1996.

[5] W. S. C. Chang, Ed., RF Photonic Technology in Optical Fiber Links. Cambridge, U.K.: Cambridge Univ. Press, 2002.

[6] S. Z. Zhang, Y. J. Chiu, P. Abraham, and J. E. Bowers, " 25 GHz polarization-insensitive electroabsorption modulators with traveling-wave electrodes," IEEE Photon. Technol. Lett., vol. 11, pp. 191-193, Feb. 1999.

[7] Y. J. Chiu, H. F. Chou, V. Kaman, P. Abraham, and J. E. Bowers, "High extinction ratio and saturation power traveling-wave electroabsorption modulator," IEEE Photon. Technol. Lett., vol. 14, pp. 792-793, June 2002.

[8] K. K. Loi, J. H. Hodiak, X. B. Mei, C. W. Tu, and W. S. C. Chang, "Linearization of 1.3- $\mu \mathrm{m} \mathrm{MQW}$ electroabsorption modulators using an alloptical frequency-insensitive technique," IEEE Photon. Technol. Lett., vol. 10, pp. 964-966, July 1998.

[9] R. B. Welstand, C. K. Sun, S. A. Pappert, Y. Z. Liu, J. M. Chen, J. T. Zhu, A. L. Kellner, and P. K. L. Yu, "Enhanced linear dynamic range property of Franz-Keldysh effect waveguide modulator," IEEE Photon. Technol. Lett., vol. 7, pp. 751-753, July 1995.

[10] W. B. Bridges and J. H. Schaffner, "Distortion in linearized electrooptic modulators," IEEE Trans. Microwave Theory Tech., vol. 43, pp. 2184-2197, Sept. 1995. 